\title{
Crystal Structure of Nanoscale Tin Dioxide Films Produced by Magnetron Sputtering
}

\author{
E.I. Sokol, O.V. Pirohov \\ Industrial and Biomedical Electronics dept. \\ National Technical University (NTU "KhPI") \\ Kharkiv, Ukraine
}

\author{
N.P. Klochko, V.A. Novikov, \\ G. S. Khrypunov, K.S. Klepikova \\ Materials Engineering for Solar Cells dept. \\ National Technical University (NTU "KhPI") \\ Kharkiv, Ukraine \\ klochko_np@mail.ru
}

\begin{abstract}
Investigation of direct current magnetron sputtering parameters effects on the crystal structure of gas sensitive tin dioxide films has revealed that the change in the substrate temperature and in the film thickness leads to the transition from the condensation of metastable conglomerates of amorphous globules to the $\approx \mathbf{1 5} \mathbf{n m}$ $\mathrm{SnO}_{2}$ crystallites with three-dimensional shape and well-defined edges. The dependence of the $\mathrm{SnO}_{2}$ structure from the working Ar- $\mathrm{O}_{2}$ gas mixtures and from their humidity evidences the significant role of the adsorption in the kinetics of the magnetron sputtering of tin dioxide. Due to the adsorption the morphological and dimensional characteristics of the tin dioxide films demonstrate the anomalous stability of the amorphous globules with their enhanced specific surface energy and the stabilization of the amorphous state, selectively retained even after the $\mathrm{SnO}_{2}$ film reach in general the critical thickness of the crystallization.
\end{abstract}

Keywords - tin dioxide; crystal structure; gas sensor; electron microscopy

\section{INTRODUCTION}

Nanoscale tin dioxide $\left(\mathrm{SnO}_{2}\right)$ layers are promising candidates for the preparing of sensing elements for metal oxide semiconductor gas sensors [1-6]. Semiconducting metal oxide thin films exhibit changes in their electrical conductivity with small amount of reducing or oxidizing gases, which provide sensors with several advantages such as low cost, short response time, wide range of target gases and high sensitivity [1, 7]. As compared with other metal oxides, tin dioxide exhibits gas sensitive properties at relatively low temperatures $\left(100-350{ }^{\circ} \mathrm{C}\right)[2,4]$, which provides a low power consumption of the sensors. Chemical durability of tin dioxide allows the use of the $\mathrm{SnO}_{2}$ based gas sensors for a detection of the majority of controlled gaseous impurities in the air including the aggressive ones. The abovementioned provides $\mathrm{SnO}_{2}$ based gas sensors with long lifetime [1-5].

Preparation of thin films by a magnetron sputtering is one of the most promising vacuum technologies for microelectronics [8-11]. This method fits well with other technological operations of modern micro- and nanoelectronics. However, a competition of the tin dioxide magnetron sputtering with a $\mathrm{SnO}_{2}$ thick film technology for the gas sensor creation and with a production of the gas sensing elements based on sintered $\mathrm{SnO}_{2}$ powders is not so successful in the production scale [2, 5-6]. A limited understanding in the literature of the mechanisms for a control of the magnetron sputtered $\mathrm{SnO}_{2}$ layers structure characteristics which determine their gas sensitivity hinder the resolution of problems encountered in their commercialization. Therefore, the investigation of the magnetron sputtering parameters effect on the crystal structure of gas sensitive tin dioxide films is relevant.

\section{EXPERIMENTAL}

Tin dioxide thin films were obtained by a direct current magnetron sputtering of a target prepared in form of a pressed $\mathrm{SnO}_{2}$ powder in $\mathrm{Ar}-\mathrm{O}_{2}$ gas atmosphere at $2 \mathrm{~Pa}$ residual pressures. The structure and phase composition of the films were investigated by X-ray diffraction analysis (XRD) on DRON-4M with a monochromatic $\mathrm{CuK}_{\alpha}$ radiation. Structural studies of the films were also carried out with a transmission electron microscope EMV-100L by the standard method. A conventional coal replica method was used for the researching of the $\mathrm{SnO}_{2}$ thin films magnetron sputtered on pyroceramic substrates. Since the tin dioxide films have a very high adhesion to the alumina ceramic substrates, their separation from these substrates and direct investigation of the film structure in a transmission electron microscope was not possible. Therefore, for their studies we have used "witnesses" on $\mathrm{NaCl}$ and mica substrates. The $\mathrm{SnO}_{2}$ films sputtered on the $\mathrm{NaCl}$ substrates were easily obtained by the salt dissolving in the water. The mica substrates were cleaved to a thickness at which $\mathrm{SnO}_{2} /$ mica becomes transparent to electrons.

Surface morphology of the obtained layers was investigated with a scanning electron microscope REM-100U in the secondary and backscattered electrons registration mode at $30 \mathrm{kV}$ accelerating voltage. Since the samples were mainly high resistivity, so the surface of the $\mathrm{SnO}_{2}$ samples was coated by vacuum deposited conductive layers to prevent the accumulation of the electric charge, namely, $\mathrm{Au}, \mathrm{Al}$ and graphite served as coating materials that should not mask the minute details of the $\mathrm{SnO}_{2}$ microstructure. The thickness of the conductive $\mathrm{Au}, \mathrm{Al}$ or graphite layer was determined for the achieving the layer with electrical continuity.

\section{RESUlts AND DISCUSSION}

Our studies have shown that magnetron sputtered tin dioxide layers deposited at $50 \%$ oxygen partial pressure in argon atmosphere onto relatively little heated substrates 


\section{IEEE XXXIV International Scientific Conference Electronics and Nanotechnology (ELNANO)}

$\left(T_{\text {sub }}=100^{\circ} \mathrm{C}\right)$ are amorphous immediately after their magnetron sputtering. This is evidenced by both absorption contrast in the electron microscopic picture and by the presence of a halo on the electron diffraction pattern (Fig. 1a). The amorphous layers do not have atomically smooth surface and consist of flakes with average size of $15 \mathrm{~nm}$. A distinctive feature of these structures is the lack of intimate contact between, no regular geometric shapes and no sharp outlines. Such $\mathrm{SnO}_{2}$ films represent conglomerates consisting of smaller particles (amorphous globules) with dimensions of about $1-2 \mathrm{~nm}$. This feature of their shape is highlighted in the schematic Fig. 1b. It can be assumed that the observed flake formation is an example of the coagulation accretion of the particles [5], when the mechanical association into a conglomerate of the amorphous globules that contact with each other occurs without their complete transformation into a spherical particle. In this case, the films are metastable not only due to its amorphous structure but also because of their relatively high specific surface area. In this case the change of the surface to volume ratio during the transition from the insular condensation step to the continuous layer formation occurs only due to the appearance of common joints between grains. So, the decrease in the surface free energy is negligible. There are plenty of cavities (pores) inside the conglomerates, which can contain adsorbed residual gases. A cause of the loosely arranged conglomerates, apparently, is a low vacuum (near $2 \mathrm{~Pa}$ ) required for the magnetron sputtering. Therefore, the atomizing gas adsorbed on the surface probably prevents the coalescence.

Tin dioxide layers deposited at higher substrate temperature $\left(T_{\text {sub }}=300{ }^{\circ} \mathrm{C}\right)$ consist of flat-shaped particles (Fig. 1c). This is evidenced by the homogeneous blackening within each particle. These particles have curved contours with more regular shape in comparison with amorphous flaks obtained on the little heated substrates. Particle sizes $(15 \mathrm{~nm})$ roughly correspond to the dimensions of the previously described conglomerates represented in Fig. 1a, b.

Electron diffractions of the samples obtained at $T_{\text {sub }}=300{ }^{\circ} \mathrm{C}$ detected continuous Debye rings and blurred diffuse halo (Fig. 1c inset), indicating the simultaneous presence of finely crystalline and amorphous components of the $\mathrm{SnO}_{2}$ condensate in these samples.

Sometimes, within a single flat particle the elements with sizes much smaller than the characteristic dimensions of the flat particles are visible contrastingly. This phenomenon can be explained by a block structure of the flat particles. The abovementioned diffraction contrast is caused by the electron diffraction in the individual crystalline blocks of the flat particles. In this case, unlike the little heated conglomerates consisting of amorphous globules isolated one from another by layers of the adsorbed atomizing gases, the flat particles are composed of disoriented but packed crystalline blocks having the same size as that of amorphous globules in the little heated films.

Part of the blocks apparently was unable to crystallize, that explains the presence of amorphous phase in these samples. That is, in this case we can see a selective crystallization inside the globules, which not leads to the formation of a monolithic crystallite throughout the volume of the original conglomerate.
Increasing the temperature of the substrate apparently leads, on the one hand, to enhanced surface migration of emerging particles and to their liquid like coalescing. On the other hand, the temperature growth reduces adsorption of the residual $\mathrm{Ar}$ and $\mathrm{O}_{2}$ gases on the surface of amorphous globules that stimulates their fusion with formation of the block particles. All this leads to a substantial reduction in the surface free energy of the particles as compared with the little heated conglomerates of globules and to the crystallization of the blocks, which form flat particles.
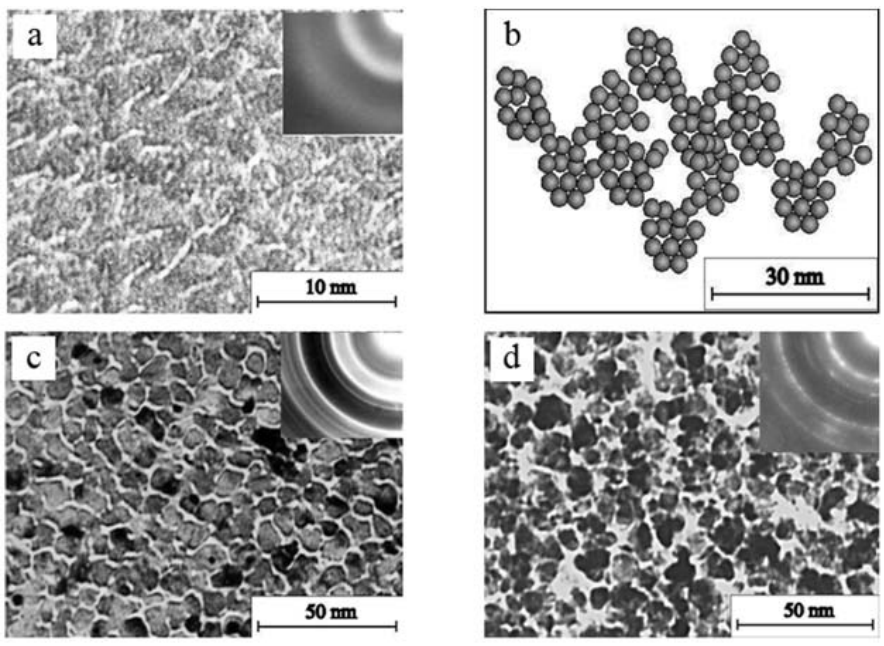

$$
\mathrm{a}, \mathrm{b}-\mathrm{T}_{\text {sub }}=100{ }^{\circ} \mathrm{C} ; \mathrm{c}-\mathrm{T}_{\text {sub }}=300{ }^{\circ} \mathrm{C} ; \mathrm{d}-\mathrm{T}_{\text {sub }}=400{ }^{\circ} \mathrm{C}
$$

Fig. 1. Effect of the substrate temperature Tsub on the tin dioxide films structure shown by electron microscopy images and electron diffraction patterns (insets)

Raising of the substrate temperature to $400{ }^{\circ} \mathrm{C}$ leads to a formation of crystalline tin dioxide layers (Fig. 1d). The obtained $\mathrm{SnO}_{2}$ crystallites in this case have a threedimensional shape and well-defined edges as compared with the particles in Fig. 1c, and their dimensions in the film plane are similar to the size of the conglomerates in the little heated films. Diffraction contrast image shows (Fig. 1d inset) single block crystalline grains; there is an absence of individual units within the majority of the crystallites. Therefore, the coherent scattering regions (approximately $15 \mathrm{~nm}$ ) in such films coincide well with sizes of the isolated particles. Electron diffraction indicates the absence of the amorphous phase in these films. Inhomogeneous distribution of intensity along the rings in the electron diffraction images indicates the appearance of texture in the $\mathrm{SnO}_{2}$ condensate, which can be attributed to the beginning of intensive coalescence.

As it was discovered during the magnetron sputtering of the gas sensitive $\mathrm{SnO}_{2}$ films on the pyroceramic substrate with continuous control of their conductivity $\sigma$, the phenomenon of a sharp increase in $\sigma$ (in several times, so-called $\Delta \sigma$ jump) when $\mathrm{SnO}_{2}$ layer reaches a certain critical film thickness $h_{c r}$ was observed. Electron microscopy, electron diffraction and X-ray diffraction studies of the films with different thicknesses grown on the $\mathrm{NaCl}$ single crystal and separated from the substrate, have shown that $\mathrm{SnO}_{2}$ films with thicknesses $h<h_{c r}$ are amorphous, while if the film thicknesses $h>h_{c r}$, then $\mathrm{SnO}_{2}$ layer contained both amorphous and crystalline phases (Fig. 2). 

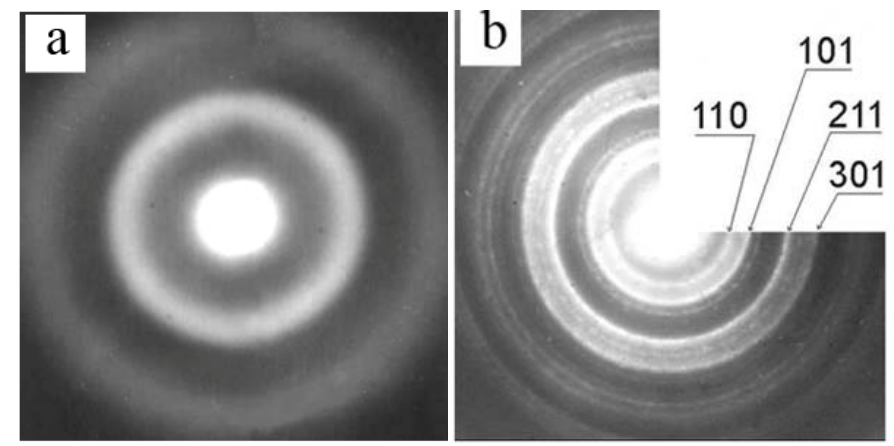

Fig. 2. Electron diffraction patterns for tin dioxide samples with different $\mathrm{SnO}_{2}$ film thicknesses: $h<h_{c r}(\mathrm{a}) ; h>h_{c r}$ (b)

These data suggest that the size effect crystallization is manifested [12], which consists in the fact that when the thickness of the amorphous films exceeds $h_{c r}$ a phase relaxation occurs, which expressed in their partial crystallization. Feature of the phase relaxation in these films is its size-selectivity, namely, preservation of the amorphous state in a certain part of the globules.

We explain this effect of selective stabilization of nonequilibrium phase by the impurity-dimensional factor [12]: the concentration of impurity gases adsorbed on $\mathrm{SnO}_{2}$ surface and their influence increase with decreasing of $\mathrm{SnO}_{2}$ particle size. Experimentally, we have found that the oxygen gas concentration $\mathrm{C}_{\mathrm{O} 2}$ in the $\mathrm{Ar}-\mathrm{O}_{2}$ sputtering gas has a significant impact on $h_{c r}$. The dependences $h_{c r}\left(T_{s u b}\right)$ for different values of $\mathrm{C}_{\mathrm{O} 2}$ are shown in Fig. 3. Furthermore, the presence of water vapor in the $\mathrm{Ar}-\mathrm{O}_{2}$ gas mixture with $C_{\mathrm{O} 2}=50 \%$, which fed into the magnetron sputtering working volume results in the $h_{c r}$ increase up to $100-150 \mathrm{~nm}$ for the films grown at $T_{s}=$ $250{ }^{\circ} \mathrm{C}$ compared with $h_{c r} \approx 70 \mathrm{~nm}$ when the $\mathrm{Ar}-\mathrm{O}_{2}$ gas mixture was subjected to the conventional purification from water vapor impurities by means of silica gel filter.

Electron microscopic studies of replicas obtained from tin dioxide films with thickness $h_{c r}$, when condensation process was stopped immediately after the jump in electrical conduction of the growing $\mathrm{SnO}_{2}$ film were fulfilled. They showed (Fig. 4a) an occurrence of "openwork" structure characteristic elements on the surface of these films, which are typical for an explosive crystallization [12]. A high rate of the electrical conductivity change ( $\Delta \sigma$ jump occurs in less than 0.01 seconds) when the film thickness equal $h_{c r}$ also confirms this phenomenon. Comparing the morphology of these surface "openwork" patterns (Fig. 4a) with morphology of the completely crystallized $\mathrm{SnO}_{2}$ film (Fig. 4b) we interpret these patterns as chains of the crystallized particles with size $L=20$ $-30 \mathrm{~nm}$ each particle, that cause the change in the electrical properties of thin films. The observed phenomenon of the explosive partial crystallization is initiated by a size factor and includes an element of randomness, so it may explain a partial irreproducibility of the electrical properties of tin dioxide films taking place even at a very strict control of the parameters during their magnetron sputtering.

The X-ray analysis of $\mathrm{SnO}_{2}$ films with a thickness in several times exceeding $h_{c r}$ indicates a simultaneous presence of amorphous and crystalline phases. The crystalline phase is in a highly dispersed state (all tested films have averaged coherent scattering regions less than $30 \mathrm{~nm}$ ). Comparison of the results of $\mathrm{X}$-ray diffraction and electron microscopic analysis reveals the block structure of crystalline particles in these $\mathrm{SnO}_{2}$ films. The block sizes are determined by the magnetron sputtering parameters. With the increase in $T_{s u b}$ during $\mathrm{SnO}_{2}$ condensation the sizes of the coherent scattering regions increase and the content of the amorphous phase in the samples decreases. Thus, the substrate temperature stimulation can explain observed crystallization and recrystallization processes during film growth. The humidity of the atomizing gases, on the contrary, leads to the formation of tin dioxide condensate with smaller coherent scattering regions and with increased content of the amorphous phase, indicating this factor as a hindrance for the crystallization process during $\mathrm{SnO}_{2}$ magnetron sputtering.

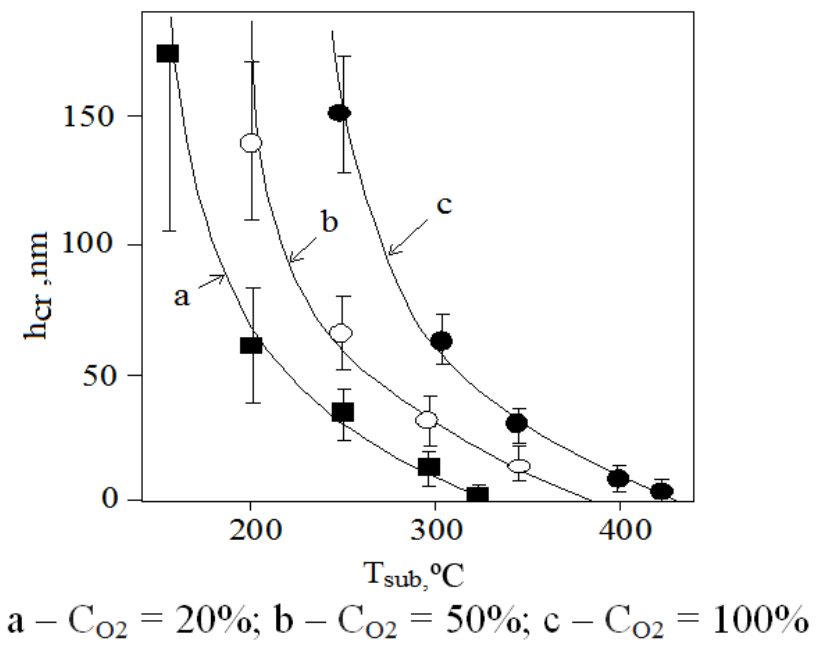

Fig. 3. Dependence of the critical $\mathrm{SnO}_{2}$ film thickness $h_{c r}$ on the substrate temperature $T_{s u b}$ for the different oxygen concentrations $C_{O 2}$ in the dry $\mathrm{Ar}-\mathrm{O}_{2}$ gas mixture (2 $\mathrm{Pa}$ total gas pressure)

By means of XRD analysis the presence of a certain amount of suboxides $\left(\mathrm{SnO}, \mathrm{Sn}_{2} \mathrm{O}_{3}, \mathrm{Sn}_{3} \mathrm{O}_{4}\right)$ was detected on the background of the main tin dioxide phase in the samples with $h>h_{c r}$. As it turned out the $450 \mathrm{~nm}$ thick films, which contained the greatest amount suboxides $C_{x}$ were obtained at $T_{\text {sub }}=200{ }^{\circ} \mathrm{C}$ or at $T_{\text {sub }}=250{ }^{\circ} \mathrm{C}$ depending on the concentration of oxygen in the gas mixture (Fig. 5). The amount of suboxides in the magnetron sputtered $\mathrm{SnO}_{2}$ films decreases with the increasing of oxygen gas concentration in the atomizing $\mathrm{Ar}-\mathrm{O}_{2}$ gas mixture.
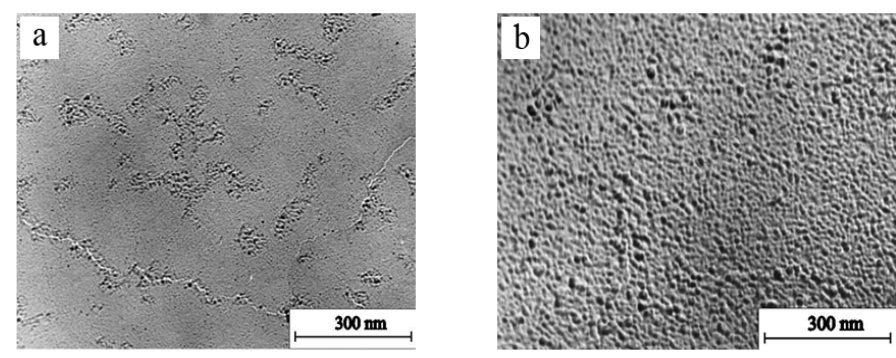

Fig. 4. Electron microscopic image of carbon replicas obtained on $\mathrm{SnO}_{2}$ films with thicknesses $h \approx h_{c r}$ (a) and $h>>h_{c r}$ (b) 


\section{IEEE XXXIV International Scientific Conference Electronics and Nanotechnology (ELNANO)}

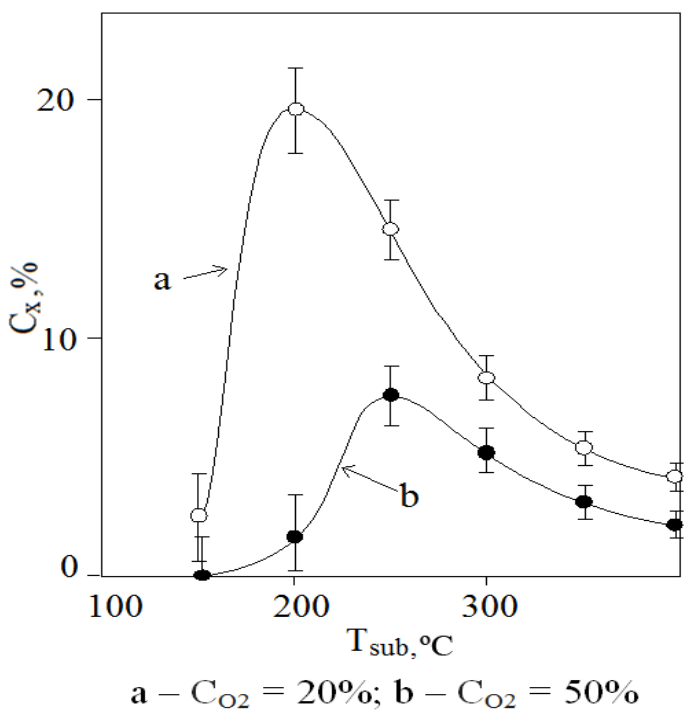

Fig. 5. Influence of the substrate temperature $T_{s u b}$ on the relative amount of suboxides $C_{x}$ in the tin dioxide films obtained by magnetron sputtering at the different oxygen gas concentrations in the atomizing $\mathrm{Ar}-\mathrm{O}_{2}$ gas mixture (2 $\mathrm{Pa}$ total gas pressure)

As such as in accordance with the model of inter-grain potential barrier [6-7], the gas sensing mechanism of metal oxide semiconductor gas sensors depends on the surface reaction between chemisorbed gases, so the prepared $\mathrm{SnO}_{2}$ films necessarily need to be stable and must have a surface with a large number of adsorption centers, be nanocrystalline and single phase to the extent possible. In the case of direct current magnetron sputtering of $\mathrm{SnO}_{2}$ target in $\mathrm{Ar}-\mathrm{O}_{2}$ gas atmosphere the above requirements are met by the following conditions: substrate temperature $T_{\text {sub }}=400{ }^{\circ} \mathrm{C}$, oxygen concentration $C_{O 2}$ more than $50 \%$, tin dioxide thickness exceeds in some times value $h_{c r}$, at which abrupt increasing of the film conductivity ( $\Delta \sigma$ jump) occurs.

\section{CONCLUSIONS}

Direct current magnetron sputtering of nanoscale tin dioxide films demonstrates that the change in the substrate temperature and in the film thickness leads to the transition from the condensation of metastable conglomerates of amorphous globules to the $\approx 15 \mathrm{~nm} \mathrm{SnO}_{2}$ crystallites with three-dimensional shape and well-defined edges.

Mode change in a relatively narrow substrate temperature interval undergoes a transition from the condensation of metastable conglomerates of amorphous globules to the $\approx 15 \mathrm{~nm} \mathrm{SnO}_{2}$ crystallites with three-dimensional shape and well-defined edges.

The dependence of the $\mathrm{SnO}_{2}$ structures from the working Ar- $\mathrm{O}_{2}$ gas mixtures and from their humidity evidences the significant role of the adsorption in the kinetics of the magnetron sputtering of tin dioxide. Due to adsorption the morphological and dimensional characteristics of the tin dioxide films demonstrate the specific thermodynamic state, which manifests itself in the anomalous stability of the amorphous globules with their enhanced specific surface energy and the stabilization of the amorphous state, selectively retained even after the $\mathrm{SnO}_{2}$ film reach in general the critical thickness of the crystallization.

\section{REFERENCES}

[1] X. Liu, S. Cheng, H. Liu, S. Hu, D. Zhang and H. Ning, "A survey on gas sensing technology," Sensors, vol. 12, pp. 9635-9665, July 2012.

[2] H. Gu, Z. Wang and Y. Hu, "Hydrogen gas sensors based on semiconductor oxide nanostructures," Sensors, vol. 12, pp. 5517-5550, April 2012.

[3] J. Gonga, Q. Chena, W. Fei and S. Seal, "Micromachined nanocrystalline $\mathrm{SnO} 2$ chemical gas sensors for electronic nose," Sensors and Actuators, vol. B 102, pp. 117-125, June 2004.

[4] G.E. Patil. D.D. Kajale, D.N. Chavan, N.K. Pawar, P.T. Ahire, S.D. Shinde, V.B. Gaikwad and G.H. Jain, "Synthesis, characterization and gas sensing performance of $\mathrm{SnO} 2$ thin films prepared by spray pyrolysis,” Bull. Mater. Sci., vol. 34, No. 1, pp. 1-9, February 2011.

[5] N. Yamazoe, "Toward innovations of gas sensor technology," Sensors and Actuators, vol. B 108, pp. 2-14, February 2005.

[6] A.S. Garde, "LPG and NH3 Sensing Properties of SnO2 Thick Film Resistors Prepared by Screen Printing Technique," Sensors \& Transducers Journal, vol. 122, Issue 11, pp. 128-142, November 2010.

[7] N.H. Al-Hardan, M.J. Abdullah, A. Abdul Aziz, "Sensing mechanism of hydrogen gas sensor based on RF-sputtered $\mathrm{ZnO}$ thin films," International journal of hydrogen energy, vol. 35, pp. 4428-4434, May 2010.

[8] A.S. Reddy, N.M. Figueiredo and A. Cavaleiro, "Nanocrystalline $\mathrm{Au}: \mathrm{Ag}: \mathrm{SnO} 2$ films prepared by pulsed magnetron sputtering," Journal of Physics and Chemistry of Solids, vol. 74, pp. 825-829, June 2013.

[9] M.A. Camacho-López, J.R. Galeana-Camacho, A. Esparza-García, C. Sánchez-Pérez and C.M. Julien, "Characterization of nanostructured $\mathrm{SnO} 2$ films deposited by reactive DC-magnetron sputtering," Superficies y Vacío, vol. 26(3), pp. 95-99, September 2013.

[10] D. Leng, L. Wu, H. Jiang, Y. Zhao, J. Zhang,W. Li, and L. Feng, "Preparation and properties of $\mathrm{SnO} 2$ film deposited by magnetron sputtering," Hindawi Publishing Corporation International Journal of Photoenergy, vol. 2012, Article ID 235971, 6 pages, June 2012.

[11] A. F. Khan, M. Mehmood, A.M. Rana, and M.T. Bhatti, "Effect of annealing on electrical resistivity of rf-magnetron sputtered nanostructured $\mathrm{SnO} 2$ thin films," Applied Surface Science, vol. 255, pp. 8562-8565, June 2009.

[12] L.S. Palatnik, M.J. Fuchs, and V.M. Kosevich, The Mechanism of Formation and Substructure of Condensed Films. Mockow: Nauka, 1972. 